\title{
Internet of Things (IoT) in Agriculture - Selected Aspects
}

\author{
M. Stočes, J. Vaněk, J. Masner, J. Pavlík
}

Department of Information Technologies, Faculty of Economics and Management, Czech University of Life Sciences Prague, Czech Republic

\begin{abstract}
Article analyzes chosen aspects of Internet of Things (IoT) in general and in regards to its specific uses in agriculture, which is one of the areas where IoT is commonly implemented. It serves as a primary delve into the issues of IoT as part of the grant received from Internal Grant Agency of Faculty of Economics and Management at CULS Prague called "Potential use of the Internet of Things, with emphasis on rural development and agrarian sector". Article overviews IoT equipment categorization, platforms, standards and network solutions. It focuses on network infrastructure, which is the foundation for IoT implementation. The specific environmental conditions of Czech Republic are also taken into account. Lastly, basic development trends of IoT are defined.
\end{abstract}

\section{Keywords}

Internet of Things, IoT, sensor, Precision Agriculture, Smart Agriculture, standards, protocols, networks.

Stočes, M., Vaněk, J., Masner, J. and Pavlík, J. (2016) "Internet of Things (IoT) in Agriculture - Selected Aspects", AGRIS on-line Papers in Economics and Informatics, Vol. 8, No. 1, pp. 83 - 88. ISSN 1804-1930. DOI: 10.7160/aol.2016.080108.

\section{Introduction}

IoT is an environment where objects, animals or people are equipped with unique identifiers capable of data transmission over Internet network without the need for human-human or humancomputer interaction (Gluhak et al., 2011). According to Juniper Research (Juniper Research, 2015) over 13,4 billion device were connected to the internet as part of IoT in 2015 and there is expected increase by $185 \%$ to 38,5 billion devices by year 2020 . IoT has uses is almost every area of modern society (Vermesan and Friess, 2013). Among the major areas are Smart Health Care, Smart Cities, Smart Industry, Autonomous Vehicles, Smart Agriculture, Precision Agriculture, Smarth Homes and others (Shang et al., 2015; Khan et al., 2012) - see figure 1.

IoT has great potential and is one of the key areas for future development of internet services. Major IT companies and most countries are keen to explore IoT issues. New uses of IoT are being searched for and established, but most of the effort is in the area of solution standardization (Jazayeri et al., 2015). Issues of IoT are part of the Digital Agenda for Europe (European Commission, 2016). As part of European research and innovation framework HORIZON 2020, it is expected that over 140 billion EURO will be invested in technologies connected to IoT just between 2016 and 2017 (European Commission, 2015). In March 2015 European Commission initiated creation of Alliance of Internet of Things Innovation (AIOTI). The goal of this alliance is to establish close cooperation between European Commission, stakeholders and parties related to IoT on innovation and standardization of IoT practices (European Commission, 2016). In Czech Republic, there is no conception that would deal with issues of IoT on government level.

One of the major problems today is the fractured nature of platforms and communication protocols which leads to incompatibility issues between various IoT devices and between different areas where IoT is implemented (Al-Fuquaha et al., 2015; Atzori et al., 2010). In precision agriculture IoT is well established, but only proprietary solution are being deployed, which leads to issues with compatibility and connection between different devices (Ojha et al., 2015; Vermesan et al., 2013). There is need to search for new solutions which would use devices compatible based on open standards and platforms. Many devices that are at least partially based on open hardware are now on the market with prices significantly lower than proprietary solutions (Fisher et al., 2015). 


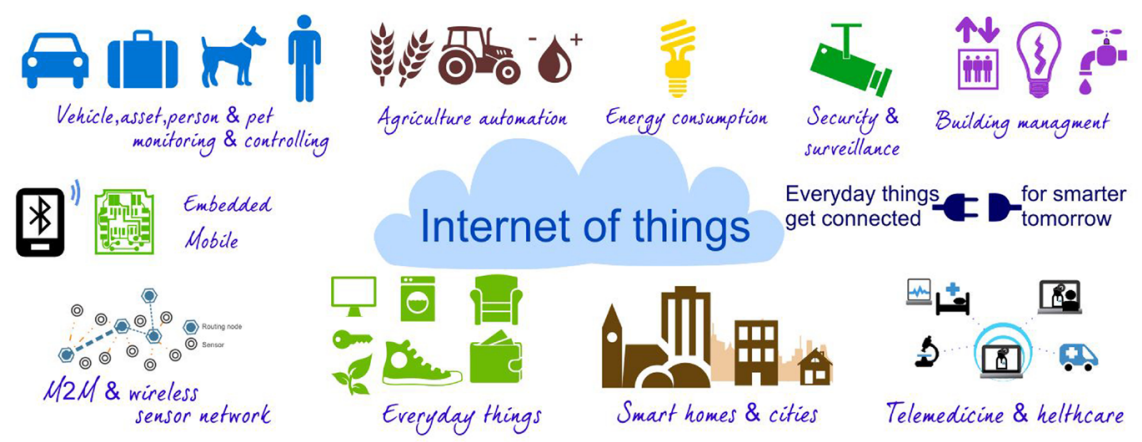

Source: http://www.engineersgallery.com

Figure 1: IoT fields.

\section{Materials and methods}

Article analyzes issues of modern IoT methods and usage in general and in the area of agriculture and serves as a base analysis study for the grant given by Internal Grant Agency of Faculty of Economics and Management (FEM) at Czech University of Life Sciences Prague (CULS). Main goal is to analyze current state of IoT and its potential in areas of rural development and agriculture in Czech Republic. This endeavor follows long term research goals of Department of Information Technologies at FEM CULS Prague and establishes new research opportunities based on current trends. It aims to compile methodic approaches, where the most important ones in terms of project solution are:

- defining and evaluating selected platforms suitable for use in IoT

- $\quad$ analyzing standards and protocols applicable in IoT

- categorization and classification of devices being used in IoT

- analyzing specifics if IoT usage in agrarian sector and similar fields

- specifying trends and opportunities for development of IoT in agrarian sector and rural development

- synthesizing obtained knowledge and proposing process of confirmation by establishing prototypes of model solution for both software and hardware part of IoT

The aforementioned represents defining of suitable technological platforms usable in IoT as well as classifying IoT devices into categories, defining and exploring implementation trends in IoT with regards to agriculture and specifics of Czech Republic environmental conditions. Prototype application solution will be developed as part of the project to determine the usability of various methodic approaches. These approaches represent a broad view upon the dynamic developing field of IoT. Research on such scale have not yet been conducted and put to use in Czech Republic. Yielded results should fill the information gaps in this area. Research is mainly focused on analyzing currently used methods and tools in IoT for data transfer and processing in agricultural sector. Experimental evaluation will test the actual impact of problematic issues for purposes of usage in agriculture. Main practical benefit of this research will be the identification of technological and technical limitations of IoT based on evaluation of actual real world implementation.

\section{Results and discussion}

\section{IoT protocols, platforms and standards}

Issues of IoT are currently being focused on by many companies, which see this area as a potential for future growth. This leads to emergence of new platforms and proprietary solutions. Compatibility of such devices is problematic which lead to creation of many alliances that focus on dealing with compatibility issues and are trying to enforce their solution and technologies for general usage (Intel ${ }^{\circledR}$ Internet of Things Solutions Alliance ... ). Apart from commercial solutions there are many community efforts trying to take advantage of open source software and open hardware. (Mesas-Carrascosa et al., 2015)

One of the examples of utilizing open hardware and software in IoT in agriculture is project Farm Bot (FarmBot, 2016), which focuses on creating humanity's open-source automated precision farming machine - see Figure 2. 


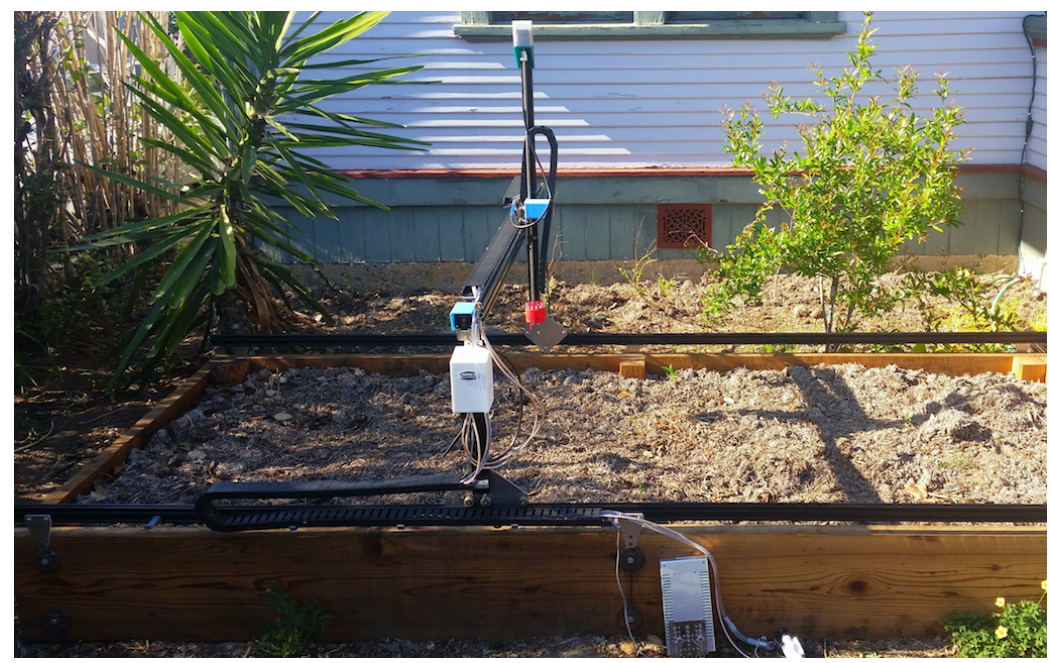

Source: https://farmbot.io/

Figure 2: FarmBot.

Most of IoT devices are based on Single Board Computer concept, which possesses necessary computing power, satisfactory dimensions, is easily expandable and utilizes open source solutions while having low energy requirements. These devices are generally based on ARM processor architecture (Advanced Reduced instruction set computing Machine). Specially developed versions on operating systems exist for these devices for both Linux and MS Windows platforms.

\section{IoT networks}

Connection to the internet is a base necessity of proper IoT device operation, in many casing such connection is wireless. Connection technologies that being deployed use various standards and can be classified based on several distinct parameters. The most common classifications for wireless connections are based on:

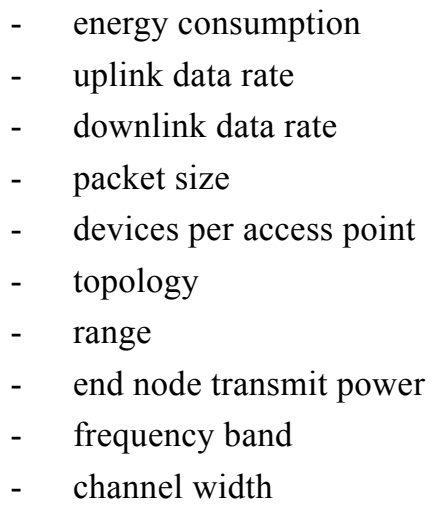

IoT comprises of many technologies that very originally developed for different purpose like GSM, LTE, Bluetooth, Wi-Fi but also uses many technologies and networks specifically designed for use in IoT. Those are for instance SigFox, LoRaWAN, IEEE P802.11ah (low power Wi-Fi), Dash 7 Alliance Protocol 1.0, RPMA, nWave (Quinnell, 2015). The main characteristic of network specifically designed for IoT usage is their low energy consumption. It is estimated that new technologies will be able to operate for many years maybe even decades using only simple battery. That is because unlike older technologies where the data transfer was the most energy demanding, newer devices have much lower consumption in that area and the most energy consuming part of the device is the sensor itself.

Figure 3 represents topology of IoT network using LoRaWAN (LoRa Alliance, 2016) technology. Data is obtained from devices using gateway which forwards it to the cloud, where it is stored and then transferred to end users through API. This communication model allows for secure and relatively easily expandable network. The main disadvantage of this type of solution is the time delay necessary for the data to be stored in cloud after being obtained (for instance from thermal sensors) and the final data usage or display. This delay can be very limiting and in some cases can render this solution completely unusable (building security etc.).

The fundamental requirement of IoT usage is network infrastructure. Due to historical development there is significant difference between Czech Republic and other developed in terms of high speed internet infrastructure (both cable and wireless). For purposes of IoT development a specialized infrastructure has to be established. 


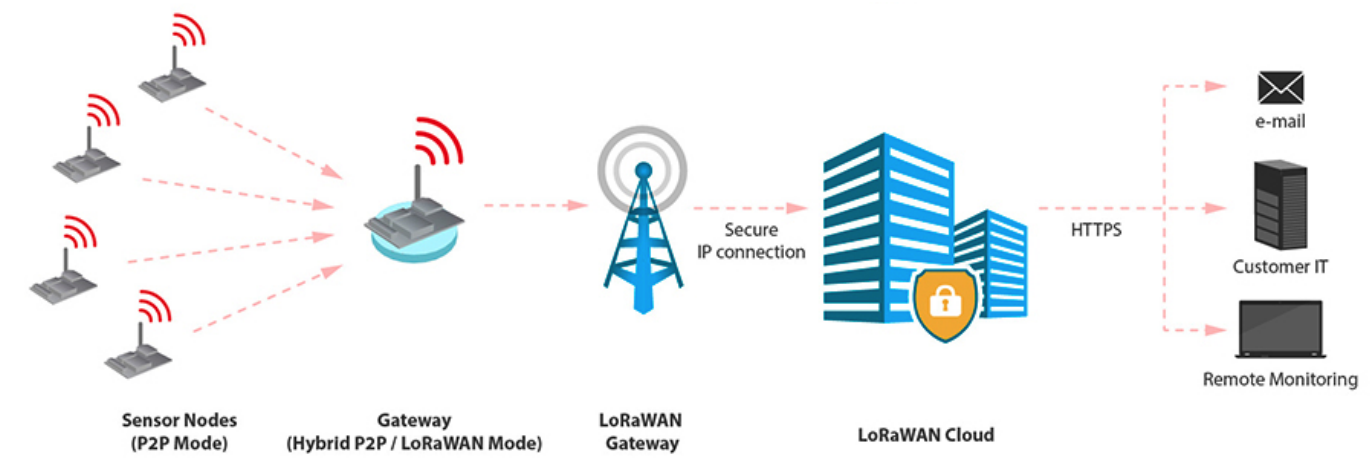

Source: https://www.cooking-hacks.com/

Figure 3: LoRaWAN schema.

At present time, three alternative networks are being established in Czech Republic that are specifically focused on servicing IoT devices. Company Czech Radio Communications is building a wireless network based on LoRa technology (České Radiokomunikace, 2016). Mobile operator T-Mobile is building a SigFox based network (Pospíšil, 2015) in cooperation with SimpleCell Networks. Third subject is Things Ltd, which is building a LoRaWAN technology based network with help of local internet service providers. The last named network might become partially free of charge.

Networks are being constructed in non-licensed range by private sector which could results in problems similar to those of high-speed internet and outdoors Wi-Fi in many areas of Czech Republic. The absence of government level policies in this sector will likely cause Czech Republic to further lag behind other countries.

\section{IoT device classification}

In general, any device connected to the internet fall into the IoT category. With that in mind, there are many distinct devices, which can be further classified based on many criteria:

- $\quad$ purpose of use

- type of internet connection

- $\quad$ type of device (sensor, server etc.)

- device dimensions

- energy consumption

\section{IoT development trends}

IoT devices can be used in almost every area of human activity and accordingly in almost every area the issues of IoT are being dealt with. It is estimated (Juniper Research, 2015), that by 2020 approximately 38.5 billion devices will be connected to the Internet.
Current IoT trends are:

- development of network technologies specific to IoT

- $\quad$ security

- $\quad$ minimizing energy consumption (increasing longevity of devices not plugged in)

- miniaturization of devices

- device integration (for instance during manufacture, eliminating the need for retrofitting)

- creation of user friendly solutions for IoT control and settings

- development of devices based on open hardware

\section{IoT in agriculture}

Devices that are currently designated as IoT are impleted in agriculture for many years now. There are mostly proprietary solutions where devices are integrated into agricultural machinery and therefore their usage is closely linked to the machine manufacturer. Major development in this area is expected in the near future, because of many projects that focus on open solutions that would eliminate compatibility issues of proprietary devices. For instance project FarmBot or MIT Media Lab Open Agriculture Initiative ("OpenAG") both deal with these issues (Open Ag Data Alliance, 2016).

\section{Conclusion}

Internet of Things (IoT) issues are more increasingly important and experience dramatic development in many areas. Such development brings many new technological innovations as well as generated new problems. Vast quantities of IoT devices in use or still in development need to be categorized based on their usage, type, internet connection, place of implementation etc. One of the important 
places of usage is agrarian sector and countryside in general. It belongs to one of the more "traditional" areas of IoT implementation, but there is still a lot of room for further development.

Concerning IoT platforms and standards, there is a push towards open source software and also open hardware, which unlike proprietary solutions deals better with device and protocol compatibility issues. Deploying such solutions could broaden the implementation possibilities of IoT as well as decrease the implementation costs and establish stronger foundations for cooperation.

Apart from increase of amount and variety of devices in use, the major development areas of IoT are: development of network technologies specific to IoT, security, miniaturization and device integration, minimizing energy requirements, software functionality support and user friendliness, usage of open source software and open hardware devices.

The absence of government-level or at least ministry-level conception for IoT in Czech Republic will surely negatively impact further development in the area (similar to what nonexistence of broadband strategy caused in the past). The state basically resigned on conceptual solution and all activity is relegated to private sector without strategic coordination. Therefore Czech Republic is expecting to lag behind other developed countries in EU and in general. That makes current issues of IoT in dire need of more focus. Results obtained will be developed in further articles as well as forwarded to government institutions such as Czech Republic Ministry of Agriculture.

\section{Acknowledgments}

The results and knowledge included herein have been obtained owing to support from the following institutional grants. Grant No. 20161012 Internal grant agency of the Faculty of Economics and Management, Czech University of Life Sciences in Prague titled „Potential use of the Internet of Things, with emphasis on rural development and agrarian sector".

\section{Corresponding author:}

Ing. Michal Stočes

Department of Information Technologies, Faculty of Economics and Management

Czech University of Life Sciences Prague, Kamýcká 129, 16521 Prague 6 - Suchdol, Czech Republic

Phone: +42022438 2277, Email: stoces@pef.czu.cz

\section{References}

[1] Al-Fuqaha,A., Guizani, M., Mohammadi, M.,Aledhari, M. and Ayyash, M. (2015) “Internet of Things: A Survey on Enabling Technologies, Protocols, and Applications”, IEEE Communications Surveys \& Tutorials, Vol. 17 No. 4, pp. 2347-2376. ISSN 1553-877x. DOI: 10.1109/COMST.2015.2444095.

[2] Atzori, L., Iera, A. and Morabito, G. (2010) “The Internet of Things: A survey”, Computer Networks, Vol..54, No.15, pp. 2787-2805. ISSN 13891286. DOI:10.1016/j.comnet.2010.05.010.

[3] České Radiokomunikace (2016) "BUDUJEME SÍŤ PRO INTERNET VĚCí NA TECHNOLOGII LORA" České Radiokomunikace [Online]. Available: https:/www.radiokomunikace.cz/budujemesit-pro-internet-veci [Accessed: 06 February 2016].

[4] Jazayeri, M., Liang, S. and Huang, C. (2015) "Implementation and Evaluation of Four Interoperable Open Standards for the Internet of Things", Sensors, Vol. 15, No. 9, pp. 24343-24373. ISSN 1424-8220. DOI:10.3390/s150924343.

[5] European Commission (2015) “Horizon 2020 Work Programme 2016-2017: Internet of Things Large Scale Pilots”, European Commission [Online] Available: http:/ec.europa.eu/digital-agenda/ en/news/horizon-2020-work-programme-2016-2017-internet-things-large-scale-pilots [Accessed: 10 February 2016].

[6] European Commission (2016) "The Internet of Things. Digital Agenda for Europe”, European Commission" [Online] Available: https://ec.europa.eu/digital-agenda/en/internet-things [Accessed: 01 February 2016].

[7] FarmBot (2016) "FarmBot genesis", FarmBot [Online]. Available: https://farmbot.io/ [Accessed: 05 February 2016]. 
[8] Fisher, R., Ledwaba, L., Hancke, G. and Kruger, C. (2015) "Open Hardware: A Role to Play in Wireless Sensor Networks?", Sensors, Vol. 15, No. 3, pp. 6818-6844. ISSN 1424-8220. DOI: $10.3390 / \mathrm{s} 150306818$.

[9] Gluhak, A., Krc, S., Nati, M., Pfristerer, D., Mitton, N. and Razafindralambo, T. (2011) “A survey on facilities for experimental internet of things research", IEEE Communications Magazine, Vol. 49, No 11, pp. 58-67. ISSN 0163-6804. DOI: 10.1109/MCOM.2011.6069710.

[10] Juniper Research (2015) “Internet of Things' Connected Devices to Almost Triple to Over 38 Billion Units by 2020", Juniper Research [Online]. Available: http://www.juniperresearch.com/press/pressreleases/iot-connected-devices-to-triple-to-38-bn-by-2020. [Accessed: 03 February 2016].

[11] Khan, R., Khan, U., Zaheer, R. and Khan, S. (2012) "Future Internet: The Internet of Things Architecture, Possible Applications and Key Challenges. In: 2012 "10 ${ }^{\text {th }}$ International Conference on Frontiers of Information Technology. IEEE, pp. 257-260. ISBN 978-0-7695-4927-9. DOI: $10.1109 /$ FIT.2012.53.

[12] LoRa Alliance (2016) "LoRa Alliance Wide Area Networks for IoT", LoRa Allinace [Online]. Available: https://www.lora-alliance.org/ [Accessed: 06 February 2016].

[13] Mesas-Carrascosa, F. J., Verdú Santano, D., Merono, J. E., Sánchez de la Orden, M. and García-Ferrer, A. (2015) "Open source hardware to monitor environmental parameters in precision agriculture”, Biosystems Engineering, Vol. 137, pp. 73-83. ISSN 15375110. DOI: 10.1016/j.biosystemseng.2015.07.005.

[14] Ojha, T., Misra, S. and Raghuwanshi, N. (2015) "Wireless sensor networks for agriculture: The state-of-the-art in practice and future challenges", Computers and Electronics in Agriculture, No. 118, pp. 66-84. ISSN 01681699. DOI: 10.1016/j.compag.2015.08.011.

[15] Open Ag Data Alliance (2016), [Online]. Available: http://openag.io/ [Accessed: 06 February 2017].

[16] Pospíšil, A. (2015) "T-Mobile postaví sit' pro internet věci”, Mobil Mania [Online] Available: http://www.mobilmania.cz/clanky/t-mobile-postavi-sit-pro-internet-veci/sc-3-a-1331933/default. aspx [Accessed: 04 February 2016].

[17] Quinnell, R. (2015) “Low power wide-area networking alternatives for the IoT", EDN Network [Online] Available: http://www.edn.com/design/systems-design/4440343/Low-power-wide-areanetworking-alternatives-for-the-IoT [Accessed: 04 February 2016].

[18] Shang, X., Zhang, R., Hu, X. and Zhou, Q. (2015) "Design theory, modelling and the application for the Internet of Things service", Enterprise Information Systems, Vol. 10, No. 3, pp. 249-267. ISSN 1751-7575. DOI: 10.1080/17517575.2015.1075592.

[19] Vermesan, O. and Friess, P. (2013) "Internet of things: converging technologies for smart environments and integrated ecosystems", Aalborg Denmark: River Publishers. ISBN 978-87-92982-96-4. 